\title{
INFINITELY MANY IDENTITIES OF KOLBERG TYPE
}

\author{
MICHAEL D. HIRSCHHORN
}

(Received 10 December 1979; revised 6 February 1980)

Communicated by A. J. van der Poorten

\begin{abstract}
O. Kolberg has shown that if the partition generating function is split into five interlocking series, then certain algebraic relations hold among these series. We show that the same phenomenon occurs whenever the number of such series is not a power of two.
\end{abstract}

1980 Mathematics subject classification (Amer. Math. Soc.): 10 A 45.

O. Kolberg (1957) has shown, inter alia, that if

$$
P_{i}=\sum_{\substack{n>0 \\ n \equiv i \bmod 5}} p(n) q^{n}, \quad i=0,1,2,3,4,
$$

where $p(n)$ is the number of unrestricted partitions of $n$, then

$$
\begin{aligned}
P_{0} P_{4}+P_{1} P_{3}-2 P_{2}^{2} & =0, \\
P_{0} P_{2}+P_{3} P_{4}-2 P_{1}^{2} & =0, \quad \text { and } \\
3 P_{1} P_{2}-2 P_{0} P_{3}-P_{4}^{2} & =0 .
\end{aligned}
$$

It is natural to ask whether the modulus 5 is special in this regard, or whether, if the partition generating function is split with respect to an arbitrary modulus $m$, there are algebraic relations between the resulting series.

As we shall see, the modulus 5 is far from being special. Writing

$$
P_{i}=\sum_{\substack{n>0 \\ n \equiv i \bmod m}} p(n) q^{n}, \quad i=0,1, \ldots, m-1,
$$

(1) Copyright Australian Mathematical Society 1981 
we shall prove the following results:

THEOREM (1.4). For each $m$ not of the form $2^{\alpha} 3^{\beta}$ there is at least one non-trivial polynomial in $P_{0}, \ldots, P_{m-1}$, homogeneous of degree $m-1$, which, considered as a power series in $q$, is identically zero.

THEOREM (1.5). For each $m$ not a power of 2 there is at least one non-trivial polynomial in $P_{0}, \ldots, P_{m-1}$, homogeneous of degree $3(m-1)$, which, considered as a power series in $q$, is identically zero.

We also derive the (new) identity which arises in our proof of Theorem (1.5) in the case $m=3$, namely

$$
\begin{aligned}
\left(P_{0}^{2}-P_{1} P_{2}\right)\left(P_{2}^{2}-P_{0} P_{1}\right)^{2} & +\left(P_{2}^{2}-P_{0} P_{1}\right)\left(P_{1}^{2}-P_{0} P_{2}\right)^{2} \\
& +\left(P_{1}^{2}-P_{0} P_{2}\right)\left(P_{0}^{2}-P_{1} P_{2}\right)^{2}=0
\end{aligned}
$$

2

Write

$$
E(q)=\prod_{n>1}\left(1-q^{n}\right), \quad J(q)=\prod_{n>1}\left(1-q^{n}\right)^{3} .
$$

We require only the classical identities of Euler and Jacobi (see Hirschhorn (1977) for elementary proofs),

$$
E(q)=\sum_{-\infty}^{\infty}(-1)^{n} q^{\left(3 n^{2}-n\right) / 2}
$$

and

$$
J(q)=\sum_{n>0}(-1)^{n}(2 n+1) q^{\left(n^{2}+n\right) / 2}
$$

If now we write

$$
P(q)=\sum_{n>0} p(n) q^{n}
$$

it is well known that

$$
P(q)=1 / E(q)
$$

Indeed (2.5) serves as a starting point for a proof of (1.2). However, for our purpose it is convenient to start with

$$
E(q)=1 / P(q)
$$


We have

$$
E(q)=1 / P(q)=\frac{P(\omega q) P\left(\omega^{2} q\right) \cdots \cdots P\left(\omega^{m-1} q\right)}{P(q) P(\omega q) \cdots \cdots P\left(\omega^{m-1} q\right)},
$$

$\omega=e^{2 \pi i / m}$. Now

$$
P(q)=P_{0}+P_{1}+\cdots+P_{m-1},
$$

so

$$
\begin{gathered}
P(\omega q)=P_{0}+\omega P_{1}+\cdots+\omega^{m-1} P_{m-1} \\
P\left(\omega^{2} q\right)=P_{0}+\omega^{2} P_{1}+\cdots+\omega^{m-2} P_{m-1} \\
\vdots \\
P\left(\omega^{m-1} q\right)=P_{0}+\omega^{m-1} P_{1}+\cdots+\omega P_{m-1}
\end{gathered}
$$

Further, we remark that

$$
D=D(q)=P(q) P(\omega q) \cdots P\left(\omega^{m-1} q\right)
$$

is a series in powers of $q^{m}$, since

$$
D(\omega q)=D(q) .
$$

From (2.7) and (2.9) it follows that

$$
\begin{aligned}
E(q)= & \left(P_{0}+\omega P_{1}+\cdots+\omega^{m-1} P_{m-1}\right)\left(P_{0}+\omega^{2} P_{1}+\cdots+\omega^{m-2} P_{m-1}\right) \\
& \times \cdots \times\left(P_{0}+\omega^{m-1} P_{1}+\cdots+\omega P_{m-1}\right) / D \\
= & \sum_{\alpha_{0}+\cdots+\alpha_{m-1}=m-1} c\left(\alpha_{0}, \ldots, \alpha_{m-1}\right) P_{0}^{\alpha_{0} P_{1}^{\alpha_{1}}} \ldots P_{m-1}^{\alpha_{m-1}} / D .
\end{aligned}
$$

Now write

$$
E(q)=E_{0}+E_{1}+\cdots+E_{m-1},
$$

where

$$
E_{i}=\sum_{\left(3 n^{2}-n\right) / 2 \equiv i \bmod m}(-1)^{n} q^{\left(3 n^{2}-n\right) / 2}, \quad i=0,1, \ldots, m-1 .
$$

It follows from (2.11)-(2.13) and the remark following (2.9) that (2.14)

$$
E_{i}=\sum_{\substack{\alpha_{0}+\cdots+\alpha_{m-1}=m-1 \\ \alpha_{1}+2 \alpha_{2}+\cdots+(m-1) \alpha_{m-1} \equiv i \bmod m}} c\left(\alpha_{0}, \ldots, \alpha_{m-1}\right) P_{0}^{\alpha_{0}} \cdots P_{m-1}^{\alpha_{m-1}} / D .
$$

Thus, $D E_{i}$ is a polynomial in $P_{0}, \ldots, P_{m-1}$ of degree $m-1$; it is easy to check that the coefficient of $P_{0}^{m-2} P_{i}$ is non-zero, so the polynomial is non-trivial. 
Further, write

$$
J(q)=J_{0}+J_{1}+\cdots+J_{m-1}
$$

where

$$
J_{i}=\sum_{\substack{n>0 \\\left(n^{2}+n\right) / 2 \equiv i \bmod m}}(-1)^{n}(2 n+1) q^{\left(n^{2}+n\right) / 2} .
$$

Then

$$
\begin{aligned}
J_{0}+J_{1}+\cdots+J_{m-1} & =J=E^{3}=\left(E_{0}+E_{1}+\cdots+E_{m-1}\right)^{3} \\
& =\sum_{\beta_{0}+\cdots+\beta_{m-1}=3} d\left(\beta_{0}, \ldots, \beta_{m-1}\right) E_{0}^{\beta_{0}} \cdots E_{m-1}^{\beta_{m-1}},
\end{aligned}
$$

from which it follows that

$$
J_{i}=\underset{\substack{\beta_{0}+\cdots+\beta_{m-1}=3 \\ \beta_{1}+2 \beta_{2}+\cdots+(m-1) \beta_{m-1} \equiv i \bmod m}}{\sum_{m-1}} d\left(\beta_{0}, \ldots, \beta_{m-1}\right) E_{0}^{\beta_{0}} \cdots E_{m-1}^{\beta_{m-1}}
$$

By virtue of (2.14) and (2.18), we can express $D^{3} J_{i}$ as a polynomial in $P_{0}, \ldots, P_{m-1}$ of degree $3(m-1)$.

The coefficient of $E_{0}^{2} E_{i}$ in $J_{i}$ is 1 or 3 , while the coefficient of $P_{0}^{m-1}$ in $D E_{0}$ is 1 , and the coefficient of $P_{0}^{m-2} P_{i}$ in $D E_{i}$ is, as noted earlier, non-zero, so the coefficient of $P_{0}^{3 m-4} P_{i}$ in $D^{3} J_{i}$ is non-zero, and the polynomial is non-trivial.

We are now in a position to prove Theorems (1.4), (1.5).

Suppose $m$ is not of the form $2^{\alpha} 3^{\beta}$. Then there is a prime $p$ such that $p \mid m$, $(p, 24)=1$. As $i$ runs through a complete set of residues $\bmod p$, so does $24 i+1$, so for some $i, 24 i+1$ is not a square $\bmod p$. For such $i$, the congruences $(6 n-1)^{2} \equiv 24 i+1 \bmod p, \frac{1}{2}\left(3 n^{2}-n\right) \equiv i \bmod p$ and $\frac{1}{2}\left(3 n^{2}-n\right)$ $\equiv i \bmod m$ have no solution. From (2.13) it follows that for such $i$,

$$
E_{i}=0
$$

which, in view of the remarks following (2.14), yields Theorem (1.4).

Suppose now that $m$ is not a power of 2. Then there is a prime $p$ such that $p \mid m$ $(p, 8)=1$. As $i$ runs through a complete set of residues $\bmod p$, so does $8 i+1$, so for some $i, 8 i+1$ is not a square $\bmod p$. For such $i$, the congruences $(2 n+1)^{2} \equiv 8 i+1 \bmod p, \frac{1}{2}\left(n^{2}+n\right) \equiv i \bmod p$ and $\frac{1}{2}\left(n^{2}+n\right) \equiv i \bmod m$ have 
no solution. From (2.16) it follows that for such $i$,

$$
J_{i}=0 \text {, }
$$

which, in view of the remarks following (2.18), yields Theorem (1.5).

Suppose $m=3$. Then

$$
\begin{aligned}
E(q) & =\left(P_{0}+\omega P_{1}+\omega^{2} P_{2}\right)\left(P_{0}+\omega^{2} P_{1}+\omega P_{2}\right) / D \\
& =\left\{\left(P_{0}^{2}-P_{1} P_{2}\right)+\left(P_{2}^{2}-P_{0} P_{1}\right)+\left(P_{1}^{2}-P_{0} P_{2}\right)\right\} / D .
\end{aligned}
$$

It follows that

$$
D E_{0}=P_{0}^{2}-P_{1} P_{2}, \quad D E_{1}=P_{2}^{2}-P_{0} P_{1}, \quad D E_{2}=P_{1}^{2}-P_{0} P_{2} .
$$

Also

$$
\begin{aligned}
J(q)= & \left(E_{0}+E_{1}+E_{2}\right)^{3} \\
= & \left(E_{0}^{3}+E_{1}^{3}+E_{2}^{3}+6 E_{0} E_{1} E_{2}\right) \\
& +3\left(E_{0}^{2} E_{1}+E_{1}^{2} E_{2}+E_{2}^{2} E_{0}\right) \\
& +3\left(E_{0} E_{1}^{2}+E_{1} E_{2}^{2}+E_{2} E_{0}^{2}\right),
\end{aligned}
$$

so

$$
\begin{aligned}
& J_{0}=E_{0}^{3}+E_{1}^{3}+E_{2}^{3}+6 E_{0} E_{1} E_{2}, \\
& J_{1}=3\left(E_{0}^{2} E_{1}+E_{1}^{2} E_{2}+E_{2}^{2} E_{0}\right), \\
& J_{2}=3\left(E_{0} E_{1}^{2}+E_{1} E_{2}^{2}+E_{2} E_{0}^{2}\right) .
\end{aligned}
$$

From (4.4) and (4.2) we obtain

$$
\begin{aligned}
D^{3} J_{0}= & \left(P_{0}^{2}-P_{1} P_{2}\right)^{3}+\left(P_{2}^{2}-P_{0} P_{1}\right)^{3}+\left(P_{1}^{2}-P_{0} P_{2}\right)^{3} \\
& +6\left(P_{0}^{2}-P_{1} P_{2}\right)\left(P_{2}^{2}-P_{0} P_{1}\right)\left(P_{1}^{2}-P_{0} P_{2}\right) \\
D^{3} J_{1}= & 3\left\{\left(P_{0}^{2}-P_{1} P_{2}\right)^{2}\left(P_{2}^{2}-P_{0} P_{1}\right)+\left(P_{2}^{2}-P_{0} P_{1}\right)^{2}\left(P_{1}^{2}-P_{0} P_{2}\right)\right. \\
& \left.+\left(P_{1}^{2}-P_{0} P_{2}\right)^{2}\left(P_{0}^{2}-P_{1} P_{2}\right)\right\}
\end{aligned}
$$

and

$$
\begin{aligned}
D^{3} J_{2}=3\left\{\left(P_{0}^{2}-P_{1} P_{2}\right)\left(P_{2}^{2}-P_{0} P_{1}\right)^{2}+\right. & \left(P_{2}^{2}-P_{0} P_{1}\right)\left(P_{1}^{2}-P_{0} P_{2}\right)^{2} \\
& \left.+\left(P_{1}^{2}-P_{0} P_{2}\right)\left(P_{0}^{2}-P_{1} P_{2}\right)^{2}\right\}
\end{aligned}
$$

The congruence $\frac{1}{2}\left(n^{2}+n\right) \equiv 2 \bmod 3$ has no solution, so

$$
J_{2}=0 \text {. }
$$


It follows that

$$
\begin{aligned}
\left(P_{0}^{2}-P_{1} P_{2}\right)\left(P_{2}^{2}-P_{0} P_{1}\right)^{2} & +\left(P_{2}^{2}-P_{0} P_{1}\right)\left(P_{1}^{2}-P_{0} P_{2}\right)^{2} \\
& +\left(P_{1}^{2}-P_{0} P_{2}\right)\left(P_{0}^{2}-P_{1} P_{2}\right)^{2}=0
\end{aligned}
$$

\section{References}

M. D. Hirschhorn (1977), 'Polynomial identities which imply identities of Euler and Jacobi', Acta Arith. XXXII, 73-78.

O. Kolberg (1957), 'Some identities involving the partition function', Math. Scand. 5, 77-92.

School of Mathematics

University of New South Wales

Kensington, N.S.W. 2033

Australia 